\title{
Morpho-orthographic segmentation without semantics
}

\author{
Elisabeth Beyersmann ${ }^{1}$ - Johannes C. Ziegler ${ }^{1} \cdot$ Anne Castles $^{2} \cdot$ Max Coltheart $^{2}$. \\ Yvette Kezilas $^{2}$ • Jonathan Grainger ${ }^{1}$
}

Published online: 20 August 2015

(C) Psychonomic Society, Inc. 2015

\begin{abstract}
Masked priming studies have repeatedly provided evidence for a form-based morpho-orthographic segmentation mechanism that blindly decomposes any word with the mere appearance of morphological complexity (e.g., corn $+e r$ ). This account has been called into question by Baayen et al. Psychological Review, 118, 438-482 (2011), who pointed out that the prime words previously tested in the morphoorthographic condition vary in the extent to which the suffix conveys regular meaning. In the present study, we investigated whether evidence for morpho-orthographic segmentation can be obtained with a set of tightly controlled prime words that are entirely semantically opaque. Using a visual lexical decision task, we compared priming from truly suffixed primes (hunter-HUNT), completely opaque pseudo-suffixed primes (corner-CORN), and non-suffixed primes (cashew$C A S H$ ). The results show comparable magnitudes of priming for the truly suffixed and pseudo-suffixed primes, and no priming from non-suffixed primes, and therefore provide further important evidence in support of morpho-orthographic segmentation processes operating in the absence of any possible role for semantics.
\end{abstract}

Keywords Morphological processing $\cdot$ Morpho-orthographic priming $\cdot$ Pseudo-affixes $\cdot$ Masked priming

Elisabeth Beyersmann

lisi.beyersmann@gmail.com

1 Laboratoire de Psychologie Cognitive, Aix-Marseille Université and Centre National de la Recherche Scientifique, 3, place Victor Hugo, Bat. 9, Case D, 13331 Marseille Cedex 1, France

2 Department of Cognitive Science and ARC Centre of Excellence in Cognition and its Disorders, Macquarie University, Sydney, Australia
The question of how morphologically complex words are processed during word recognition has prompted many years of research and is still a hotly debated subject. One account that has become increasingly influential during the past decade is that skilled readers rapidly decompose morphologically structured letter strings (e.g., hunter) into their morphemic subunits (e.g., hunt $+e r$ ) during reading. Importantly, evidence for the morphological segmentation of letter strings has been found in "pseudo-suffixed" words that have the appearance of morphological complexity (e.g., corn $+e r$ ) but do not share the same meaning as the embedded pseudo-stem (e.g., corn vs. corner). Evidence for this account stems primarily from masked priming studies showing that the recognition of a target word (as assessed by a visual lexical decisions task) is facilitated by the prior presentation of a related suffixed (e.g., hunter-HUNT) or pseudo-suffixed prime (e.g., corner-CORN), but not by a nonsuffixed prime (e.g., cashew-CASH). This finding was initially reported by Longtin, Segui, and Hallé (2003) in French and by Rastle, Davis, and New (2004) in English-speaking participants. Since these initial studies, the basic findings have been replicated across a number of different Indo-European languages, such as Dutch (Diependaele, Sandra, \& Grainger, 2005; Diependaele, Sandra, \& Grainger, 2009) and Russian (Kazanina, 2011; Kazanina, Dukova-Zheleva, Geber, Kharlamov, \& Tonciulescu, 2008) and have had a major influence on theorizing in the field (for a review, see Rastle \& Davis, 2008).

However, a major criticism to the morpho-orthographic processing literature was put forward by Baayen, Milin, Filipovic Durdevic, Hendrix, and Marelli (2011), who drew attention to one potential significant flaw in previous morphological priming studies. They pointed out that prime words previously tested in the pseudo-morphological priming condition varied in the extent to which the suffix conveyed its regular meaning. For example, as Baayen and colleagues (2011) pointed out, the etymological origin of archer ("someone who 
wields a bow") is Latin arcus (bow, arc), which is similar in structure to a denominal formation, such as trucker ("someone who drives a truck"). More precisely, Baayen et al. (2011) suggested that priming in the pseudo-suffixed condition has previously only been observed, "because for the majority of opaque items, the suffix is fully functional in the meaning of the complex word (p. 466)." Thus the pseudo-suffixed word sniper contains the pseudo-suffix er, which carries the typical meaning of this suffix as "someone who does something." When applied to suffixes with syntactic function, such as the suffix " $y$ " (adjectival marker) in pseudo-suffixed words like "crafty," then this rule indeed disqualifies many of the items tested by Rastle et al. (2004). ${ }^{1}$

The goal of the present study was to put to test the important claims of Baayen et al. (2011) using Rastle et al.'s (2004) experimental design with more tightly controlled materials. Following Rastle et al., we carried out a masked priming lexical decision experiment comparing priming effects on stem target recognition for truly suffixed (e.g., hunter-HUNT) and pseudo-suffixed prime-target pairs (e.g., corner-CORN) relative to a nonsuffixed control (e.g., cashew-CASH). However, in contrast to the pseudo-suffixed words used by Rastle and colleagues, we used a set of revised and more stringent stimulus selection criteria in this condition to ensure that the whole word and the stem never shared any meaning, not even remotely (e.g., butcher was excluded) or archaically (e.g., archer was excluded; see Materials section for more details) and that the pseudo-suffix did not carry its meaning or function as a real suffix. If Baayen and colleagues are correct that priming in the pseudosuffixed condition has been previously observed only because for the majority of items the suffix was fully functional in the meaning of the complex word, then we expect to observe priming in the truly suffixed condition, but not in the pseudo-suffixed or non-suffixed conditions. If however, a morpho-orthographic segmentation mechanism indeed exists that blindly decomposes any word that has the mere appearance of morphological complexity, we should replicate the standard pattern of morphoorthographic priming effects. That is, we should find comparable priming in both the truly suffixed and pseudo-suffixed condition and no priming in the non-suffixed control condition.

\section{Method}

\section{Participants}

Forty students from Macquarie University, all English native speakers, participated for course credit.

\footnotetext{
${ }^{1}$ Note that although not mentioned by Baayen et al. (2011), the same criticism applies to Longtin et al.'s (2003) study in French, where, for example, the pseudo-suffix -er does have its typical function (infinitive verb) in pseudo-suffixed primes such as traiter, repasser, and soudoyer.
}

\section{Materials}

In the first instance, a list of pseudo-suffixed words (e.g., corner) was selected from the CELEX lexical database (Baayen, Piepenbrock, \& van Rijn, 1993), using a number of strict selection criteria. Pseudo-suffixed words were selected such that the whole word and the stem never shared any remote meaning (e.g., butcher was excluded) or archaic meaning (e.g., archer was excluded). We excluded words where the suffix conveyed some degree of meaning (e.g., gaffer was excluded, because although gaffer and gaff are not semantically related, a gaffer is "someone who does something"). We excluded words where suffixes conveyed a syntactic content (e.g., crafty was excluded, because adding the derivational suffix $y$ turns a word into an adjective). Moreover, we ensured that the target was always a noun, verb or adjective (e.g., offeroff and wither-with were excluded). Primes and targets that expressed colloquial, archaic or technical terms were excluded (e.g., laden-lad, bastion-bast, lander-land, and liter-lit were excluded). We also excluded low frequency primes and targets (e.g., scullion), inflected targets (e.g., sold), words containing low frequency suffixes (e.g., et as in helmet), words containing ambiguous suffixes (e.g., illion vs. ion in billion), and words containing ambiguous stems (e.g., note vs. not in notion). Finally, primes were never the orthographic or deletion neighbour of a word that was morphologically related to the stem (e.g. career was excluded, because carer is related to the stem care). The application of these criteria resulted in the selection of 22 pseudo-suffixed words, including the suffixes $-e n,-e r$, and -ion, which are listed in Table 3. Each pseudosuffixed prime (e.g., corner) was then paired with a target, which was the word embedded in the whole letter string (e.g., corner-CORN).

In addition to the list of pseudo-suffixed items, we selected a list of 22 truly suffixed and 22 non-suffixed items. The truly suffixed items were chosen such that the meaning of the prime could always be derived from the meaning of its stem-target (e.g., hunter-HUNT). Moreover, since the suffix er can be used in inflectionally (e.g., darker) and derivationally (e.g., speaker), only derivational instances of $e r$ were chosen. In the non-suffixed control condition, primes and targets were selected such that the relationship was purely orthographic (e.g., cashew-CASH). The stimuli-matching criteria were adapted from Rastle et al. (2004). All three conditions were matched on orthographic overlap (calculated by dividing prime length by target length), target neighbourhood size, target length, target frequency, prime length, and prime frequency (see Table 1 for characteristics). Word frequencies were extracted from the SUBTLEX database (Van Heuven, Mandera, Keuleers, \& Brysbaert, 2014). In addition, semantic relatedness values for each prime-target pair in all three conditions were extracted from Latent Semantic Analysis Web facility (http://lsa.colorado.edu/; Landauer \& Dumais, 1997), 
Table 1 Mean word frequencies were extracted from the SUBTLEX database (Van Heuven et al., 2014) and are given as Zipf values (log10 occurrences per billion)

\begin{tabular}{lclc}
\hline Properties & $\begin{array}{l}\text { Truly } \\
\text { suffixed }\end{array}$ & Pseudo-suffixed & Non-suffixed \\
\hline Targets & & & \\
$\quad$ Log word frequency & $4.76(0.85)$ & $4.40(0.79)$ & $4.57(0.82)$ \\
Orthographic & $8.4(5.0)$ & $12.0(6.6)$ & $9.7(5.3)$ \\
$\quad$ neighbourhood size & & & $16.9(8.2)$ \\
$\begin{array}{c}\text { Phonological } \\
\text { neighbourhood size }\end{array}$ & $18.5(8.8)$ & $19.9(8.5)$ & $3.6(0.5)$ \\
$\begin{array}{l}\text { Length, in letters } \\
\text { Length, in phonemes }\end{array}$ & $3.9(0.6)$ & $3.6(0.6)$ & $3.1(0.6)$ \\
$\begin{array}{c}\text { Primes } \\
\text { Log word frequency }\end{array}$ & $4.03(0.64)$ & $3.91(0.81)$ & $3.93(0.70)$ \\
$\begin{array}{l}\text { Length, in letters } \\
\text { Length, in phonemes }\end{array}$ & $6.1(0.8)$ & $5.9(0.6)$ & $5.9(0.6)$ \\
$\begin{array}{l}\text { Orthographic } \\
\text { prime-target overlap }\end{array}$ & $1.6(0.2)$ & $1.6(0.2)$ & $1.6(0.2)$ \\
$\quad \begin{array}{l}\text { Semantic prime-target } \\
\text { relatedness }\end{array}$ & $0.43(0.22)$ & $0.10(0.08)$ & $0.12(0.08)$ \\
\hline
\end{tabular}

Standard deviations are shown in parentheses. Orthographic neighbourhood size, phonological neighbourhood size, length (in letters and phonemes), and orthographic overlap for primes and targets were taken from the CELEX database (Baayen et al., 1993). Semantic relatedness values were calculated using the Latent Semantic Analysis Web facility (Landauer \& Dumais, 1997)

which revealed that semantic relatedness values in the truly suffixed condition (.43) were significantly higher than those in both the pseudo-suffixed $(0.10), \mathrm{t}(27)=6.64$, and the nonsuffixed $(0.12), t(27)=6.37$, conditions, but the pseudosuffixed and non-suffixed conditions did not differ, $\mathrm{t}(40)=$ 0.58 .

For each target, an orthographically, morphologically, and semantically unrelated control prime was selected from the CELEX lexical database (e.g., nearly-HUNT, faulty-CORN, seeing-CASH), which was matched to the related primes on frequency and length. Given that each target word was paired with two different types of prime (related and unrelated), two lists were created so that each target only appeared once in each list, but each time in a different priming condition. Participants were allocated to one of the two lists, such that they only ever saw any target once. A full list of stimuli is provided in the Table 3.

In line with Rastle et al. (2004), an additional set of 22 unrelated word pairs was included to further reduce the prime-target relatedness proportion. The related and unrelated prime-target pairs were matched on target length. Finally, for the purpose of the lexical decision task, we included a set of 88 nonword targets (e.g., $S A R B$ ), which were all orthographically legal and pronounceable and matched on length to the realword targets. Each nonword target was preceded by a morphologically complex word prime (e.g., novelty-SARB).

\section{Procedure}

Stimuli were presented in the centre of a LED computer screen using DMDX software (Forster \& Forster, 2003). Participants were tested individually in a quiet room. Each trial consisted of a 500-ms forward mask of hash keys, then a 50 -ms prime in lowercase, then the uppercase target. The target remained present until the response was made or until 3 seconds had elapsed. Participants were instructed to respond as quickly and accurately as possible if the visually presented items were real English words or not. Stimuli were presented in randomized order. The experiment lasted for approximately 10 minutes per participant.

\section{Results}

Lexical decisions to word targets were analysed as follows. Incorrect responses were removed from the reaction time (RT) analysis ( $7.8 \%$ of all data). Inverse RTs (1/RT) were calculated for each participant to correct for RT distribution skew and used throughout the analyses. Reaction times smaller than $\mathrm{Q} 1-(2.5 \times \mathrm{IQR})$ or larger than $\mathrm{Q} 3+(2.5 \times \mathrm{IQR})$, by either participants or items, were excluded from the analyses $(\mathrm{Q} 1=$ first quartile, Q3 = third quartile, and IQR $=\mathrm{Q} 3-\mathrm{Q} 1=$ interquartile range). This trimming led to the removal of $4.1 \%$ of the data. RTs and error rates are presented in Table 2 and were analysed for each subject.

We used linear mixed-effect modelling to perform the main analyses (Baayen, 2008; Baayen, Davidson, \& Bates, 2008). Fixed and random effects were only included if they significantly improved the model's fit in a backward stepwise model selection procedure. Models were selected using chi-squared log-likelihood ratio tests with regular maximum likelihood

Table 2 Mean lexical decision times and error rates for word targets averaged across subjects

\begin{tabular}{llll}
\hline Condition & Reaction times & Error rates & Example \\
\hline $\begin{array}{l}\text { Truly suffixed } \\
\quad \text { Related }\end{array}$ & $536(65)$ & $4.3(6.5)$ & hunter-HUNT \\
$\quad$ Unrelated & $561(72)$ & $4.5(6.5)$ & nearly-HUNT \\
$\quad$ Priming effect & 25 & 0.2 & \\
Pseudo-suffixed & & & \\
$\quad$ Related & $564(64)$ & $8.4(8.8)$ & corner-CORN \\
$\quad$ Unrelated & $587(68)$ & $10.7(10.5)$ & faulty-CORN \\
$\quad$ Priming effect & 23 & 2.3 & \\
Non-suffixed & & & \\
$\quad$ Related & $567(80)$ & $4.0(5.9)$ & cashew-CASH \\
$\quad$ Unrelated & $565(69)$ & $6.0(9.6)$ & seeing-CASH \\
$\quad$ Priming effect & -2 & 2.0 & \\
\hline
\end{tabular}

Standard deviations are shown in parentheses 
parameter estimation. Trial order was included to control for longitudinal task effects such as fatigue or habituation. Although target words and prime words were matched on logarithmic word frequency (Zipf frequency; Van Heuven et al., 2014) across item types (truly suffixed, pseudo-suffixed, non-suffixed), we included logarithmic target frequency and logarithmic prime frequency as covariates in the analyses.

A linear mixed-effects model as implemented in the lme4 package (Bates, Maechler, Bolker, \& Walker, 2014) in the statistical software R (Version 3.0.3; RDevelopmentCoreTeam, 2008) was created with five fixed effects factors (prime type: related, unrelated; item type: truly suffixed, pseudo-suffixed, non-suffixed; trial order; logarithmic target frequency; logarithmic prime frequency), their interactions, and two random effects factors (random intercepts for subjects and items). The lmer default coding for treatment contrasts was used for factor prime type (i.e., reference level "related"). Pairwise comparisons of factor item type were obtained by setting different baseline conditions within the same model (i.e. using lmer function 'relevel'). $P$ values were determined using the package lmerTest (Kuznetsova, Brockhoff, \& Christensen, 2014). The model was refitted after excluding data-points whose standardised residuals were larger than 2.5 in absolute value (1.2\%; see Baayen, 2008). Factors trial order and logarithmic prime frequency were not significant and was therefore excluded from the analyses. The linear mixed-effects model estimated pairwise level-by-level analyses for fixed-effects factor analyses, yielding three prime type by item type comparisons. RT analyses revealed a significant priming effect in the truly suffixed condition $(t=4.91, p<0.001$; less than the Bonferroni corrected value) and in the pseudo-suffixed condition $(t=3.96, p<0.001$; less than the Bonferroni corrected value). No priming was obtained in the non-suffixed condition $(t=1.20, p=0.229)$. Moreover, the results revealed that priming was significantly greater in the truly suffixed than in the non-suffixed condition $(t=2.57, p=0.010)$ and priming in the pseudo-suffixed condition was significantly greater than priming in the non-suffixed condition $(t=1.98, p=0.047)$, but there was no significant difference between the priming in the truly suffixed and pseudo-suffixed conditions $(t=0.53$, $p=0.594)$. In addition, there was a significant effect of logarithmic stem frequency $(t=6.31, p<0.001)$. No other effects were significant.

Error analyses followed the same logic as the RT analyses. We applied a binomial variance assumption to the trial-level binary data using the function glmer as part of the R-package lme4. The results revealed that participants made significantly fewer errors in the truly suffixed condition, compared to the pseudo-suffixed and non-suffixed conditions $(z=2.45$, $p=0.014 ; z=2.08, p=0.038$, respectively), but there was no difference between the pseudo-suffixed and non-suffixed condition $(z=0.38, p=0.704)$. No other effects were significant.

\section{Discussion}

The goal of the present study was to test the recent claim that previously observed pseudo-suffixed priming effects (see Rastle \& Davis, 2008, for a review) have only been obtained due to the large number of partially transparent items in this condition (such as sniper or crafty; Baayen et al., 2011). According to Baayen et al. (2011), the fact that the pseudosuffixes (-er and $-y$ in these examples) actually carry their true meaning/function in the pseudo-suffixed words (i.e., a sniper is someone who does something, crafty is an adjective) is the reason why pseudo-suffixed priming effects have been observed in prior research. In discussing how their Naive Discriminative Reader (NDR) model could simulate the statistically equivalent opaque and transparent priming effects observed by Rastle et al. (2004), Baayen et al. (2011) state that "...the reason that the transparent and opaque conditions give rise to a similar priming effect... is because orthographic representations for the suffix (unigrams, bigrams) have become associated with the suffix meaning. Crucially, these associations can emerge because for the majority of the opaque items, the suffix is fully functional in the meaning of the complex word (p. 466)."

To test this account of pseudo-suffixed priming, we conducted a masked primed lexical decision experiment comparing truly suffixed (e.g., hunter-HUNT) and pseudo-suffixed prime-target pairs (e.g., corner-CORN) relative to a nonsuffixed control condition (e.g., cashew-CASH). In contrast to previously conducted research, we used a set of revised and more stringent stimulus criteria to select our set of pseudosuffixed prime words. Most importantly, the pseudo-suffixed words were selected such that the pseudo-affix never conveyed any semantic content (e.g., er in sniper, where er means "someone who does something") or syntactic function (e.g., $y$ in crafty, where $y$ turns the word into an adjective) that matched the meaning or syntactic category of the pseudosuffixed word. The results of the present study revealed robust and equal magnitudes of priming in the truly suffixed and pseudo-suffixed conditions, while priming was absent in the non-suffixed control condition. Our findings thus unambiguously demonstrate that complex words are decomposed into morphemic subunits during the early stages of visual word recognition, independently of whether they bear a true morphological structure (e.g., hunt + er) or a pseudomorphological structure (e.g., corn + er).

The present results clearly challenge amorphous theories of visual word recognition, and in particular the NDR model of Baayen and colleagues (2011). The NDR model accounts for "morphological" phenomena observed during the processing of written language on the basis of learned associations between sublexical orthography and semantics, without any role for any kind of morpho-orthographic segmentation mechanism. Thus, in the NDR model, the orthographic 
representation of an embedded stem in truly suffixed words (e.g., hunter) is associated with the same meaning as the stem when it stands as a separate word (hunt), whereas an embedded pseudo-stem in pseudo-suffixed words (e.g., corner) is associated with a different meaning. Moreover, associations between a suffix and semantics are only implemented if the suffix conveys a regular meaning (for further discussion, see Baayen et al., 2011, pp. 465-467). Thus, for pseudo-suffixed words in which there is no synchronic suffix (as in corner), no suffix meaning is assigned. Hence, the model predicts no priming from truly pseudo-suffixed primes, or at least no difference between the pseudo-suffixed prime condition and the orthographic prime condition, which is inconsistent with the present results.

On the other hand, the equivalent magnitudes of priming in the truly suffixed and pseudo-suffixed conditions are in line with a considerable number of studies suggesting that there must be an automatic, fast-acting morpho-orthographic segmentation mechanism that rapidly decomposes morphologically complex letter strings into morphemic units, independently from semantics (Beyersmann, Castles, \& Coltheart, 2011; Diependaele et al., 2005, 2009; Duñabeitia, Perea, \& Carreiras, 2007; Rastle \& Davis, 2008; Rastle et al., 2004; Taft, 2003; Taft \& Ardasinski, 2006; Taft \& Nguyen-Hoan, 2010). It has been proposed that morpho-orthographic segmentation is guided by some kind of 'affix-stripping' mechanism (firstly introduced by Taft \& Forster, 1975), that matches input letter sequences with higher-level affix representations, while the remaining letter string activates the representation of the stem. The notion that affixes play an active role during the reading of morphologically complex words is further supported by evidence from masked affix priming studies (e.g., Crepaldi, Hemsworth, Davis, \& Rastle, 2015; Duñabeitia, Perea, \& Carreiras, 2008; Giraudo \& Grainger, 2003), suggesting that the recognition of suffixed target words is facilitated by the prior presentation of a suffixed prime which shares the same suffix (e.g., baker-WALKER).

Finally, we note that the pattern of results obtained in the present study may be specific to the lexical decision paradigm. Recent evidence suggests that morpho-orthographic segmentation might not be obligatory, as indicated by the fact that morpho-orthographic effects were not found in a masked primed semantic categorisation task (Marelli, Amenta, Morone, \& Crepaldi, 2013), nor in a same-different judgment task (Duñabeitia, Kinoshita, Carreiras, \& Norris, 2011). That is, semantically blind morphological decomposition appears to be at least partially task-dependent (Amenta, Marelli, \& Crepaldi, 2015).

In conclusion, the presented evidence is clearly inconsistent with the claim that previously reported morphoorthographic priming effects observed with the lexical decision task were only carried by the large proportion of transparent suffixes in the pseudo-morphological priming condition. Hence, our findings speak against amorphous theories of morphological processing that predict that there should be no priming from pseudo-suffixed prime stimuli.

Acknowledgments This project was supported by a postdoctoral grant from the Fyssen Foundation to Elisabeth Beyersmann. This research was carried out within the Labex BLRI (ANR-11-LABX-0036) and has benefited from support from the French government, managed by the French National Agency for Research (ANR), Investments of the Future A*MIDEX (ANR-11-IDEX-0001-02).

\section{Appendix}

Table 3 Stimuli, including truly suffixed, pseudo-suffixed, and nonsuffixed items

\begin{tabular}{|c|c|c|}
\hline \multicolumn{3}{|c|}{ Truly suffixed condition: } \\
\hline weaken & grassy & WEAK \\
\hline tighten & grocery & TIGHT \\
\hline boxer & snowy & BOX \\
\hline owner & bumpy & OWN \\
\hline farmer & boards & FARM \\
\hline player & bricks & PLAY \\
\hline banker & postal & BANK \\
\hline singer & greedy & SING \\
\hline dealer & saving & DEAL \\
\hline killer & sleepy & KILL \\
\hline hunter & nearly & HUNT \\
\hline reader & widely & READ \\
\hline leader & purity & LEAD \\
\hline teller & fruity & TELL \\
\hline mixer & rocky & MIX \\
\hline winner & lately & WIN \\
\hline tester & oddity & TEST \\
\hline abortion & sculptor & ABORT \\
\hline eviction & governor & EVICT \\
\hline fusion & poetry & FUSE \\
\hline action & sailor & ACT \\
\hline edition & robbery & EDIT \\
\hline \multicolumn{3}{|c|}{ Pseudo-suffixed condition: } \\
\hline related prime & unrelated prime & target \\
\hline pollen & fluffy & POLL \\
\hline siren & petal & SIR \\
\hline bother & acidic & ВОTH \\
\hline brother & zealous & BROTH \\
\hline cater & bushy & CAT \\
\hline corner & faulty & CORN \\
\hline cower & gawky & COW \\
\hline flower & earthy & FLOW \\
\hline lager & doing & LAG \\
\hline mister & sticky & MIST \\
\hline mother & pricey & MOTH \\
\hline
\end{tabular}


Table 3 (continued)

\begin{tabular}{|c|c|c|}
\hline ponder & syrupy & POND \\
\hline proper & bossy & PROP \\
\hline shower & smelly & SHOW \\
\hline taper & messy & TAP \\
\hline tower & milky & TOW \\
\hline wander & stormy & WAND \\
\hline legion & filthy & LEG \\
\hline lotion & lovely & LOT \\
\hline mission & eatable & MISS \\
\hline portion & wealthy & PORT \\
\hline ration & likely & RAT \\
\hline Non-suffixed condition: & & \\
\hline related prime & unrelated prime & target \\
\hline cashew & seeing & $\mathrm{CASH}$ \\
\hline fleece & arming & FLEE \\
\hline turnip & glassy & TURN \\
\hline ballast & prowess & BALL \\
\hline spinach & buttery & SPIN \\
\hline pulpit & joyous & PULP \\
\hline starve & fairly & STAR \\
\hline galaxy & asking & GALA \\
\hline freeze & ageing & FREE \\
\hline address & speaker & ADD \\
\hline tease & salty & TEA \\
\hline pillow & curled & PILL \\
\hline dragon & bubby & DRAG \\
\hline window & darker & WIND \\
\hline yellow & catchy & YELL \\
\hline carrot & sooner & CAR \\
\hline hotel & risky & HOT \\
\hline tooth & beefy & TOO \\
\hline begin & griped & BEG \\
\hline skirt & dusty & SKI \\
\hline wonder & gloomy & WON \\
\hline carton & girdle & CART \\
\hline
\end{tabular}

\section{References}

Amenta, S., Marelli, M., \& Crepaldi, D. (2015). The fruitless effort of growing a fruitless tree: Early morpho-orthographic and morpho-semantic effects in sentence reading. Journal of Experimental Psychology: Learning, Memory and Cognition, Advance online publication.

Baayen, R. H. (2008). Analyzing linguistic data: A practical introduction to statistics using $R$. Cambridge: Cambridge University Press.

Baayen, R. H., Piepenbrock, R., \& van Rijn, H. (1993). The CELEX lexical database (CD-ROM). Philadelphia: Linguistic Data Consortium, University of Pennsylvania.

Baayen, R. H., Davidson, D. J., \& Bates, D. M. (2008). Mixed-effects modeling with crossed random effects for subjects and items. Journal of Memory and Language, 59, 390-412.

Baayen, R. H., Milin, P., Filipovic Durdevic, D., Hendrix, P., \& Marelli, M. (2011). An amorphous model for morphological processing in visual comprehension based on naive discriminative learning. Psychological Review, 118, 438-482.

Bates, D., Maechler, M., Bolker, B., \& Walker, S. (2014). Ime4: Linear mixed-effects models using Eigen and S4. R package version 1.1-5. http://CRAN.R-project.org/package=lme4

Beyersmann, E., Castles, A., \& Coltheart, M. (2011). Early morphological decomposition during visual word recognition: Evidence from masked transposed-letter priming. Psychonomic Bulletin \& Review, 18(5), 937-942.
Crepaldi, D., Hemsworth, L., Davis, C. J., \& Rastle, K. (2015). Masked suffix priming and morpheme positional constraints. The Quarterly Journal of Experimental Psychology, Advance online publication, $1-16$.

Diependaele, K., Sandra, D., \& Grainger, J. (2005). Masked cross-modal morphological priming: Unravelling morpho-orthographic and morpho-semantic influences in early word recognition. [Article]. Language and Cognitive Processes, 20(1-2), 75-114.

Diependaele, K., Sandra, D., \& Grainger, J. (2009). Semantic transparency and masked morphological priming: The case of prefixed words. Memory \& Cognition, 37, 895-908.

Duñabeitia, J. A., Perea, M., \& Carreiras, M. (2007). Do transposed-letter similarity effects occur at a morpheme level? Evidence for morphoorthographic decomposition. [Article]. Cognition, 105(3), 691-703.

Duñabeitia, J. A., Perea, M., \& Carreiras, M. (2008). Does darkness lead to happiness? Masked suffix priming effects. Language and Cognitive Processes, 23, 1002-1020.

Duñabeitia, J. A., Kinoshita, S., Carreiras, M., \& Norris, D. (2011). Is morpho-orthographic decomposition purely orthographic? Evidence from masked priming in the same-different task. Language \& Cognitive Processes, 26, 509-529.

Forster, K. I., \& Forster, J. C. (2003). DMDX: A Windows display program with millisecond accuracy. Behavior Research Methods, Instruments, \& Computers, 35(1), 116-124.

Giraudo, H., \& Grainger, J. (2003). On the role of derivational affixes in recognizing complex words: Evidence from masked priming. In R. H. Baayen \& R. Schreuder (Eds.), Morphological structure in language processing (pp. 209-232). Berlin: Mouton de Gruyter.

Kazanina, N. (2011). Decomposition of prefixed words in Russian. Journal of Experimental Psychology: Learning, Memory, and Cognition, 37(6), 1371-1390.

Kazanina, N., Dukova-Zheleva, G., Geber, D., Kharlamov, V., \& Tonciulescu, K. (2008). Decomposition into multiple morphemes during lexical access: A masked priming study of Russian nouns. Language \& Cognitive Processes, 23(6), 800-823.

Kuznetsova, A., Brockhoff, P. B., \& Christensen, R. H. B. (2014). ImerTest: Tests for random and fixed effects for linear mixed effect models (lmer objects of lme4 package): R package version 2.0-6. http://CRAN.R-project.org/package=lmerTest

Landauer, T. K., \& Dumais, S. T. (1997). A solution to Plato's problem: The latent semantic analysis theory of acquisition, induction, and representation of knowledge. Psychological Review, 104, 211-240.

Longtin, C. M., Segui, J., \& Hallé, P. A. (2003). Morphological priming without morphological relationship. Language and Cognitive Processes, 18(3), 313-334.

Marelli, M., Amenta, S., Morone, E. A., \& Crepaldi, D. (2013). Meaning is in the beholder's eye: Morpho-semantic effects in masked priming. Psychonomic Bulletin \& Review, 20(3), 534-541.

Rastle, K., \& Davis, M. H. (2008). Morphological decomposition based on the analysis of orthography. Language \& Cognitive Processes, 23, 942-971.

Rastle, K., Davis, M. H., \& New, B. (2004). The broth in my brother's brothel: Morpho-orthographic segmentation in visual word recognition. Psychonomic Bulletin \& Review, 11, 1090-1098.

RDevelopmentCoreTeam. (2008). $R$ : A language and environment for statistical computing: $\mathrm{R}$ package version 1.1-5. http://CRAN.Rproject.org/package $=1$ me 4

Taft, M. (2003). Morphological representation as a correlation between form and meaning. In E. Assink \& D. Sandra (Eds.), Reading complex words (pp. 113-137). Amsterdam: Kluwer.

Taft, M., \& Ardasinski, S. (2006). Obligatory decomposition in reading prefixed words. The Mental Lexicon, 1(2), 183-199.

Taft, M., \& Forster, K. I. (1975). Lexical storage and retrieval of prefixed words. Journal of Verbal Learning and Verbal Behavior, 14, 638-647. 
Taft, M., \& Nguyen-Hoan, M. (2010). A sticky stick? The locus of morphological representation in the lexicon. Language \& Cognitive Processes, 25(2), 277-296.
Van Heuven, W. J. B., Mandera, P., Keuleers, E., \& Brysbaert, M. (2014). Subtlex-UK: A new and improved word frequency database for British English. The Quarterly Journal of Experimental Psychology, 67, 1176-1190. 